\title{
Resumé of the challenges and burden of living with transplanted hearts for $>31$ years
}

\author{
Eva Maria Javier Delmo ${ }^{1}$, Mariano Francisco del Maria Javier ${ }^{2}$, Frank Wagner ${ }^{1}$, Roland Hetzer ${ }^{2}$ \\ ${ }^{1}$ Universitätsmedizin Berlin-Charité, Charité Research Organization, Berlin, Germany; ${ }^{2}$ Department of Cardiothoracic and Vascular Surgery, Cardio \\ Centrum Berlin, Berlin, Germany \\ Correspondence to: Eva Maria Javier Delmo, MD, MSc, PhD. Universitätsmedizin Berlin-Charité, Charité Research Organization, Virchowweg 10, \\ 10117 Berlin, Germany. Email: eva.javierdelmo@gmail.com.
}

\begin{abstract}
Heart transplantation has extended the lives of many patients with end-stage heart failure. Although beset with the arduous aftermaths of immunosuppression, those who survived live meaningful lives similar to that of the general population. In this series, heart transplantation has led to a considerable extension of life span of >31-34 years. Development of cardiac allograft vasculopathy is a major concern in the longterm follow-up of transplant patients. Immunosuppressive drugs have a major impact on the development and progression of cardiac allograft vasculopathy and the main cause of cardiac allograft loss after the first posttransplantation year. Post-transplant neoplasia remains a challenging long-term problem for patients after orthotopic heart transplantation. Post-transplant lymphoproliferative disease appears to be largely the result of the effectiveness of current agents used for long-term immunosuppression, and the neoplasms typically harbor the Epstein Barr virus genome, which is presumed to play a major role in neoplastic transformation. Posttransplantation, new diseases may occur or there may be worsening of pre-existing diseases. By the end of the $5^{\text {th }}$ year, all suffered from hypertension. Renal insufficiency developed within 5 years after transplantation, and $10 \%$ have a creatinine value of $>2.5 \mathrm{mg} / \mathrm{dL}$. Hyperlipidemia, mostly hypercholesterolemia occurs especially in patients on tacrolimus or everolimus. Quality of life is not impaired despite somatic problems. In this period $>31$ years post-post-orthotopic heart transplantation, they even reported a surprising increase in their emotional well-being. The 10 patients are active and satisfied with their daily lives. They regard their own life as meaningful, have good families and active social relations. Nonetheless, it is burdened by chronic allograft vasculopathy and immunosuppression sequelae, the main limiting factors for survival, which needed to be constantly addressed throughout their lifetime. This brief report provides an insight into the challenges and burden patients living $>31$ years with transplanted hearts are confronted with.
\end{abstract}

Keywords: Chronic transplant allograft vasculopathy; orthotopic heart transplantation (OHT); immunosuppression; post-transplantation lymphoproliferative disease; quality of life; re-transplantation

Submitted Feb 25, 2020. Accepted for publication Aug 20, 2020.

doi: $10.21037 / \mathrm{cdt}-20-286$

View this article at: http://dx.doi.org/10.21037/cdt-20-286

\section{Introduction}

Survival after orthotopic heart transplantation (OHT) is excellent, particularly if it is compared with the natural course of end-stage heart failure (ESHF). The most recent data of the registry of the International Society of Heart and Lung Transplantation (ISHLT) involving 114,783 heart transplantations from January 1992 to June 2017 indicates a current median year survival of 11.9 years (1).
This has improved, albeit slight, as compared to the median survival of 10 years $(n=78,050)$ with 122 patients at risk in 22 years, based on the 1982-2007 registry of the ISHLT. The development of new immunosuppressive drugs which allow a variety of immunosuppressive regimens, tailored to the individual patient, has contributed to this improvement, since rejection and the adverse sequelae of immunosuppression has been better controlled. After 
20 years, approximately $21 \%$ of patients are still alive, according to the international registry (1). In some experienced centers, long-term survival is reported to be even higher $(2,3)$. Whether heart transplant recipients transplanted at centers with superior short-term outcomes live longer on long-term follow-up remains unreported (4).

Only few papers with patients surviving more than 20 years after OHT have been reported $(2,3,5-8)$. There are only small cohorts, and therefore the clinical characteristics associated with prolonged survival are not very well understood. There are sporadic single reports on patients living for 30 to 33 years after OHT, albeit published as newspaper articles. Detailed information on cardiac allograft vasculopathy (CAV) and the immunosuppression sequelae in these patients is largely unknown to the scientific community. No series to date has described any transplantation recipients with more than 30 years of follow-up.

This report provides a resumé of the burden and challenges the patients, who are still living $>31$ years after OHT, are affronted with.

\section{Overview of the 10 heart-transplanted patients living for $>31$ years}

From the first orthotopic heart transplantation in 1986 until August 2014 in Berlin, 1804 heart transplantations (plus the 70 patients transplanted in Hannover from 1983-1985) (9), which overall summed up to 1874) have been carried out including 52 re-transplantations but excluding the 101 combined heart and lung transplantations. Generally, all transplanted patients are strictly advised to have routine follow-up including laboratory tests, echocardiography, endomyocardial biopsy when necessary and coronary angiography. In the first year post-OHT, the patients are monitored monthly. In the second until the fifth year post-OHT, quarterly medical examinations per year are mandatory. After the fifth year post-OHT, follow-ups are conducted once annually. Thereafter, the regular posttransplantation follow-up is being done by the respective local attending physicians who then refer the patients back to us accordingly.

Of 147 transplants performed between February 1, 1985 and September 30,1987, 10 patients survived longer than 31 years post-OHT and are the main focus of this report. Their medical records including operative procedures and follow-up reports were thoroughly reviewed.

Two of these patients are taking a combination of cyclosporine and prednisolone while six are taking a combination of cyclosporine, azathioprine and prednisolone. One patient takes cyclosporine, azathioprine and everolimus and another one takes a combination of cyclosporine, azathioprine and tacrolimus. Nine patients are taking antihypertensive drugs, six are taking ASA or platelet aggregation inhibitors and all are taking diuretics.

Clinical data on the last follow-up which includes general physical status based on New York Heart Association (NYHA) classification, cardiovascular (coronary artery status, left ventricular ejection fraction, valve function) performance and renal behavior, occurrence of any malignancy, onset of CAV, progression of existing diseases, and current medications being taken were obtained from the attending physicians' medical reports.

Information on psychosocial status was carried out through personal conversations with each patient and questionnaires modified from several existing quality of life (QoL) assessment standards (10-12) to evaluate psychosomatic complaints, subjective limitations experienced by exhaustion tendency, gastric complaints, limb pain and the global score of discomfort, perceived social support, health-related QoL with regards to physical functions, somatic pains, overall health, vitality, social relations, emotional and mental health.

\section{Case series}

The Institutional Review Board approved this study. The informed consent requirement was waived as the study itself was regarded as non-interventional and data protection standards were fulfilled.

Table 1 shows the profile of the 10 patients ( 2 females) living for more than 31 years after OHT. Median age of these patients at heart transplantation was 23.4 (range, 4.6-49.3). Median age during the last follow-up (October 10,2019 ) was 56.0 (range, 36.9-80.9).

Four patients (ages 55.5, 50.7, 77.4 and 71.3 years old) are still living after $>34$ years, 1 ( 70.3 years old) after $>33$ years, another $4(70.8,55.9,36.9$ and 56.8 , years old $)$ after 32 years and 1 ( 80.9 years old) after $>31$ years.

The course of each patient's life post-OHT, with the burdens associated with living with a transplanted heart for a long-time is briefly described below.

\section{Living >34 years after heart transplantation}

The first patient (male, now 55.5 years old) developed 


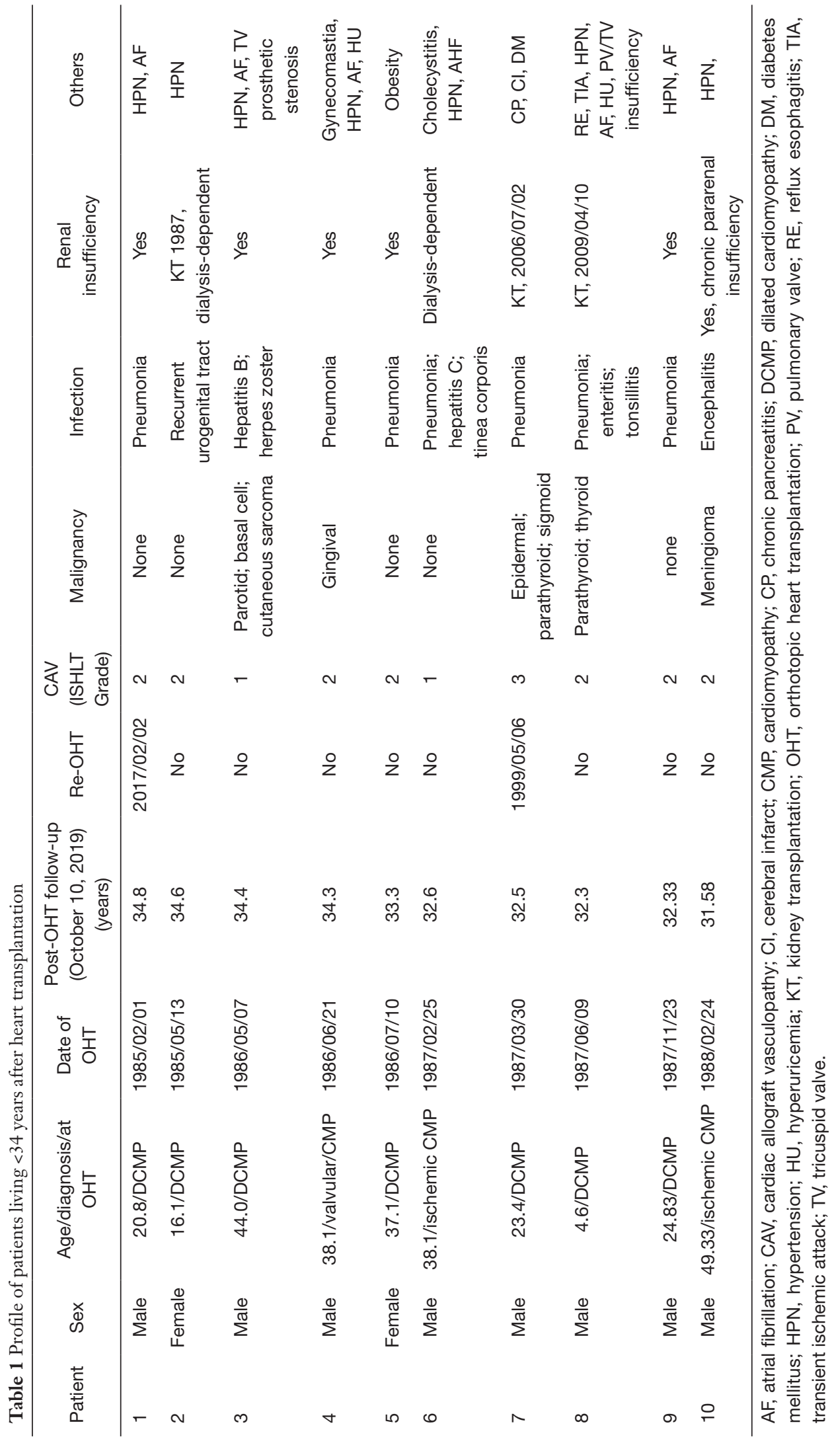


recurrent pneumonia and chronic renal insufficiency. Through the years, he has been encumbered with hypertension and atrial fibrillation. These, however, did not prevent him from managing a landscaping business. $\mathrm{He}$ underwent re-transplantation for CAV 32 years after the primary OHT. No malignancy has developed. He has been happily married and is engaged in many social activities.

The second patient (female, now 50.6 years old) has recurrent urogenital tract infections, developed renal failure and had been dialysis-dependent until she underwent renal transplantation 2 years post-OHT. Except from having hypertension, she has no other co-morbidities. No malignancy has developed. She is employed and happily married.

The third patient (male, now 77.4 years old) underwent tricuspid valve replacement with Hancock $33 \mathrm{~mm} 10$ years after the primary OHT. He developed several malignancies, i.e., parotid carcinoma (cylindroma, 6 years after OHT), skin tumors (basal cell carcinoma and cutaneous sarcoma, 31 years post-OHT), which were all treated surgically. He had shown signs of chronic allograft rejection 13 years post-OHT which was successfully managed medically. He underwent herniotomy (20 years post OHT) and cholecystectomy (23 years post-OHT). He had recurrent herpes zoster infections and had acquired Hepatitis B. He developed chronic renal insufficiency, hypertension, atrial fibrillation and hyperuricemia. During the latest follow-up it was shown that his tricuspid valve prothesis has become stenotic. He eventually underwent surgery for removal of a prosthetic thrombus. He has retired from work and enjoying his quiet retirement with his family.

The fourth patient (male, now 71.3 years old) developed gingival carcinoma 2 years post-OHT, for which he underwent surgery. He had recurrent pneumonia and developed renal disease. He has hypertension, atrial fibrillation and hyperuricemia. Over the post-OHT years, he developed gynecomastia. He has been retired from work and enjoying his life in the countryside.

\section{Living $>33$ years after heart transplantation}

This patient [female, now 70.3 years old, transplanted due to ESHF from dilated cardiomyopathy (DCMP)] showed signs of allograft rejection 2 years post-OHT which was successfully treated medically. She had recurrent bouts of pneumonia and developed renal insufficiency and obesity. No malignancy has developed. She has retired from work and continue spending quality times with her family.

\section{Living $>32$ years after heart transplantation}

The first patient (male, now 70.6 years old, transplanted due to ESHF from ischemic cardiomyopathy) had recurrent bouts of pneumonia, had acquired Hepatitis C and afflicted with recurrent tinea corporis. He had severe renal insufficiency and became dialysis dependent. He had cholecystitis and suffering from hypertension and atrial fibrillation. No malignancy has developed. He is enjoying his retirement years and engages himself in various social activities.

The second patient (male, now 55.9 years old) developed transplant vasculopathy and underwent re-heart transplantation 12 years after the primary OHT and a coronary artery bypass grafting a year later. He developed 3 malignancies, i.e., epidermal (intraepidermal squamous cell carcinoma, 22 years post-OHT), parathythyroid (12 years post-OHT) and sigmoid (21 years post-OHT), for which surgeries were performed. He had recurrent bouts of pneumonia. He underwent kidney transplantation for severe renal failure 19 years post- OHT. He suffers from chronic pancreatitis, cerebral infarct and developed diabetes mellitus. He is currently employed and living with his family.

The third patient (male, now 36.9 years old) developed parathyroid and thyroid carcinoma 21 years after primary OHT und underwent surgery for these. He had recurrent bouts of pneumonia, tonsillitis and enteritis. A year later (22 years post-OHT), he underwent kidney transplantation, had signs of rejection managed medically. He suffers from reflux esophagitis, had transient ischemic attacks, hypertension, hyperuricemia and atrial fibrillation. During the last followup, he was found to have tricuspid and pulmonary valve insufficiency. He is currently employed, married and has two children.

The fourth patient (male, now 56.8 years old) had recurrent bouts of pneumonia and developed chronic renal insufficiency, hypertension and atrial fibrillation. No malignancy has developed. He is currently employed, married and has 2 children.

\section{Living >31 years after heart transplantation}

This patient (male, now 80.9 years old) had meningioma a year post-OHT which was resected. with subsequent encephalitis which was then resolved. He also showed signs of allograft rejection successfully abated with medical management. He then developed chronic pararenal 
insufficiency and hypertension. He has been in retirement for over 15 years, still physically active, and enjoying his life with his wife, children and grandchildren.

\section{Functional status}

The NYHA functional class shows overall a good physical status of the patients. Eight patients are in NYHA functional class I with a normal systolic left ventricular ejection fraction (>55\%) and 2 are in NYHA functional class II with ejection fractions of $45-50 \%$. No patient is classified to be in NYHA functional class III or IV.

\section{Cardiac status}

Interestingly, only one of these 10 patients underwent a 3 -vessel coronary artery revascularization for coronary artery stenosis one year after his re-transplantation. All the other seven patients with CAV (ISHLT grade 2) underwent interventional coronary procedures while the other 2 with CAV (ISHLT grade 1) are being closely monitored regarding progression of CAV.

As shown in Table 1, all developed cardiac allograft vasculopathy of different severity based on ISHLT classification.

Six patients had pathological changes in their ECG rhythm which was atrial fibrillation. Normal function of all heart valves was found in 8 patients while mild to moderate tricuspid and pulmonary valve insufficiency was found in one patient A tricuspid valve replacement was implanted in one patient.

\section{Renal status}

All patients had various degrees of renal dysfunction, which is a consequence of immune suppression. Two had underwent renal transplantation, and one is hemodialysisdependent, as described in the case series.

\section{Burdens and challenges}

\section{Late graft-associated diseases and sequelae of immunosuppression therapy}

\section{Transplant vasculopathy}

Development of CAV is a major concern in the longterm follow-up of transplant patients. Immunosuppressive drugs have a major impact on the development and progression of cardiac allograft vasculopathy and the main cause of cardiac allograft loss after the first post-transplantation year. Localized or focal stenosis was diagnosed in all 10 patients within 5-15 years post-OHT. Interventional procedures were done on those with ISHLT grade 2. CAV was the main cause for retransplantation in a patient after a post-OHT time of 12.25 years. It has been well documented that the early onset of CAV is a predictor of rapid development of severe forms of $\mathrm{CAV}$, while the patients with late onset $\mathrm{CAV}$ have a delayed onset of CAV.

\section{Post-transplant lymphoproliferative disease (PTLD)}

Post-transplant neoplasia remains a challenging longterm problem for patients post-OHT. PTLD appears to be largely the result of the effectiveness of current agents used for long-term immunosuppression, and the neoplasms typically harbor the Epstein Barr virus (EBV) genome, which is presumed to play a major role in neoplastic transformation.

Development of malignancy occurred at different times, within 3-20 years post-OHT. Skin and mucosal cancer had developed in 3 patients, such as basal cell carcinoma, cutaneous sarcoma and intraepidermal squamous cell carcinoma. One patient had developed gingival carcinoma. Three patients even developed several malignancies in different organs of the body (parotid glands, thyroid, parathyroid, sigmoid, brain). Five patients have not developed any malignancy at all.

\section{Other associated diseases}

Post-transplantation, new diseases may occur or there may be worsening of pre-existing diseases. In the first year after transplantation, 4 patients developed hypertension and by the end of the $5^{\text {th }}$ year, all suffered from hypertension.

Renal insufficiency developed among all 10 patients within 5 years after transplantation, and $10 \%$ have a creatinine value of $>2.5 \mathrm{mg} / \mathrm{dL}$. At this time, 4 patients required chronic dialysis and eventually 3 underwent renal transplantation. The renal insufficiency that occur postOHT is dependent on cyclosporin A, as well as arterial hypertension and atherosclerosis.

Hyperlipidemia, mostly hypercholesterolemia occurs in all patients especially in patients on tacrolimus or everolimus. One patient in this series developed diabetes mellitus 5 years post-OHT. This occurs in approximately $4-5 \%$ of patients on cyclosporin therapy and in $10-15 \%$ of 
patients on tacrolimus.

Remarkable is the susceptibility of these patients to recurrent bacterial infections and infections of viral etiologies.

\section{Psychosocial data}

Heart transplantation enabled these patients with previous terminal heart failure to get back to a "normal" life. Based on their responses to the questionnaires, quality of life was reduced to a certain acceptable degree.

\section{Anxiety and depression}

Noteworthy was that all these 10 patients reported having neither pathological anxiety nor depression symptoms.

\section{Social relations}

The 10 patients expressed gratifying satisfaction with their social relations and support over the years post-OHT.

\section{Quality of life}

When these 10 patients were asked how they regard their overall health, 7 of them answered with "good to excellent" and "less good"/satisfactory by 3. Not one of them assessed their overall health as "bad." Most of them experienced exhaustion tendency, gastric disturbances, pain in the extremities and joints, from time to time; however, they viewed them as part of the normal aging process and do not limit their daily routine activities.

When they were asked about their perception of the quality of their lives with regards to physical, social and environment, 6 of them responded with highly satisfactory, 2 of them with satisfactory and another 2 as decently fine.

Quality of life is not impaired despite somatic problems. In this period $>31$ years post-OHT, they even reported a surprising increase in their emotional well-being. The 10 patients are active and satisfied with their daily lives. They regard their own life as meaningful, have good families and active social relations.

\section{Conclusions}

Heart transplantation currently remains the treatment of choice for patient with severely advanced heart failure. No other treatment options, such as medical therapy or electrical and mechanical devices, can compete with the excellent results, particularly over the long-term.

In this series, heart transplantation has led to a considerable extension of life span of $>31-34$ years. Be it as it may, it is burdened by chronic allograft vasculopathy and immunosuppression sequelae, the main limiting factors for survival, which needed to be constantly addressed throughout their lifetime. Considering the tremendous advances in the immunosuppressive therapy and in the understanding of CAV pathophysiology, we can hope for even better results in the next years.

\section{Acknowledgments}

Funding: None.

\section{Footnote}

Provenance and Peer Review: This article was commissioned by the editorial office, Cardiovascular Diagnosis and Therapy for the series "Heart Failure in the Young and Old: Insights into Various Therapies". The article has undergone external peer review.

Conflicts of Interest: The authors have completed the ICMJE uniform disclosure form (available at http://dx.doi. org/10.21037/cdt-20-286). The series "Heart Failure in the Young and Old: Insights into Various Therapies" was commissioned by the editorial office without any funding or sponsorship. RH served as the unpaid Guest Editor of the series, and serves as an unpaid editorial board member of Cardiovascular Diagnosis and Therapy from Jul 2019 to Jun 2021. The authors have no other conflicts of interest to declare.

Etbical Statement: The authors are accountable for all aspects of the work in ensuring that questions related to the accuracy or integrity of any part of the work are appropriately investigated and resolved. All procedures performed in studies involving human participants were in accordance with the ethical standards of the institutional and/or national research committee(s) and with the Helsinki Declaration (as revised in 2013). Individual consent was waived as the study itself was regarded as non-interventional and data protection standards were fulfilled.

Open Access Statement: This is an Open Access article distributed in accordance with the Creative Commons Attribution-NonCommercial-NoDerivs 4.0 International License (CC BY-NC-ND 4.0), which permits the noncommercial replication and distribution of the article with 
the strict proviso that no changes or edits are made and the original work is properly cited (including links to both the formal publication through the relevant DOI and the license). See: https://creativecommons.org/licenses/by-nc-nd/4.0/.

\section{References}

1. Khush KK, Cherikh WS, Chambers DC, et al. The International Thoracic Organ Transplant Registry of the International Society for Heart and Lung Transplantation: Thirty-sixth adult heart transplantation report - 2019; focus theme: Donor and recipient size match. J Heart Lung Transplant 2019;38:1056-66.

2. Deuse T, Haddad F, Pham M, et al. Twenty-year survivors of heart transplantation at Stanford University. Am J Transplant 2008;8:1769-74.

3. Mastrobuoni S, Dell'Aquila AM, Azcarate PM, et al. Longterm survival (>20 years) following heart transplantation. J Cardiovasc Surg (Torino) 2012;53:677-84.

4. Singh TP, Mehra MR, Gauvreau K. Long-Term Survival After Heart Transplantation at Centers Stratified by ShortTerm Performance. Circ Heart Fail 2019;12:e005914.

5. Jaramillo N, Segovia J, Gómez-Bueno M, et al. Characteristics of patients with survival longer than 20

Cite this article as: Javier Delmo EM, Javier MFDM, Wagner F, Hetzer R. Resumé of the challenges and burden of living with transplanted hearts for $>31$ years. Cardiovasc Diagn Ther 2021;11(1):164-170. doi: 10.21037/cdt-20-286 years following heart transplantation. Rev Esp Cardiol (Engl Ed) 2013;66:797-802.

6. Ongcharit $\mathrm{P}$, Wongkietkachorn K, Sritangsirikul S, et al. Heart transplantation 1987--2007: 20 years' experience at Chulalongkorn hospital. Transplant Proc 2008;40:2591-3.

7. Roussel JC, Baron O, Périgaud C, et al. Outcome of heart transplants 15 to 20 years ago: graft survival, posttransplant morbidity, and risk factors for mortality. J Heart Lung Transplant 2008;27:486-93.

8. Roussel JC, Baron O, Périgaud C, et al. Outcome of heart transplants 15 to 20 years ago: graft survival, posttransplant morbidity, and risk factors for mortality. J Heart Lung Transplant 2008;27:486-93.

9. Hetzer R, Warnecke H, Schüler S, Süthoff U, Borst HG. Heart transplantation--a two-year experience. Z Kardiol 1985;74 Suppl 6:51-8.

10. Development of the World Health Organization WHOQOL-BREF quality of life assessment. The WHOQOL Group. Psychol Med 1998;28:551-8.

11. Bullinger M, Kirchberger I. SF-36. Fragebogen zum Gesundheitszustand. Handanweisung. Göttingen: Hogrefe, 1998.

12. Zigmond AS, Snaith RP. The hospital anxiety and depression scale. Acta Psychiatr Scand 1983;67:361-70. 